\title{
Wind Turbine Aerodynamic Performance by Lifting Line Method
}

\author{
HORIA DUMITRESCU* ${ }^{*}$ and VLADIMIR CARDOS ${ }^{\dagger}$ \\ Institute of Applied Mathematics, Romanian Academy, Calea 13 Septembrie 13, P.O. Box 1-24, RO-70700 Bucharest, Romania
}

(Received 15 November 1996; In final form 13 February 1997)

\begin{abstract}
The vortex model of propellers is modified and applied to the high-speed horizontal axis turbines. The turbine blades are replaced by lifting lines and trailing vortices which shed along the blade span. The model is not a free wake model, but it is still a nonlinear one which should be solved iteratively. In addition to the regular case where the trailing vortices are constrained to distribute along a helical surface, another version, where each trailing vortex sheding from the blade grows as a free helical vortex line, is also included. Performance parameters are calculated by application of the Biot-Savart law along with the KuttaJoukowski theorem. Predictions are shown to compare favorably with existing numerical data from more involved free wake methods, but require less computational effort. Thereby, the present method may be a very useful tool for calculating the aerodynamic loads on horizontal-axis wind turbine blades.
\end{abstract}

Keywords: Wind power, Wind turbines, HAWT-rotors, Blade aerodynamics, Lifting line theory, Incompressible flows

\section{INTRODUCTION}

With a rise of interest in wind energy, intensive research on the aerodynamic behavior of wind turbines has been conducted during the past decades. Methods concerned with the prediction of aerodynamic loading and performance of wind turbines have been reviewed in De Vries [1979] and De Vries [1983]. Even now there are only a few theoretical analyses which are not based on the momentum theory Gohard [1978], Miller [1983], Maekawa [1984], Afjeh and Keith [1986].
It has been commonly agreed that the key to an accurate calculation of the rotor aerodynamic behavior is the correct modelling of the rotor wake. Because of the very complex structure of the wake it was also evident that purely analytical methods are limited and one should turn to numerical methods in order to obtain general solutions.

Classification of the methods is based on the manner in which the wake is modelled and the induced velocity at a blade section is evaluated. There are two main approaches to the problem of wake modelling. The first method is known as the

* Corresponding author.

$\dagger$ Tel.: 0040-1-4104082. Fax: 0040-1-3354305. E-mail: vcardos@iacob.ima.ro or vcardos@tyr.racai.ro. 
"prescribed wake" or "rigid wake" (PWM). According to this method the geometry of the wake is known a priori, which implies that the velocity field, or rather an approximation to it, has been assumed. Once the wake geometry has been prescribed, the corresponding induced velocity and circulation distributions along the blade can be calculated. The geometry of the wake is determined by using different kinds of assumptions, while in most of the cases these assumptions are based on experimental evidence. The second method is the "free-wake" analysis (FWM). In this method an initial geometry of the vortex wake is assumed. The wake is regarded as being composed of a large number of discrete vortex elements, and these elements are allowed to convect in the velocity field they create. Provided the numerical method employed is convergent, the vortex elements will move until they take up positions which are consistent with the velocity field (wake in equilibrium state). As might be expected, the computer requirements for such calculations are prodigious, which makes this kind of analysis somewhat impractical as a design tool. This is the reason why some investigators have divided the wake into two or three different regions (near, intermediate and far wake), at each region the computations being done in appropriate way to that region. This approach causes somewhat reduction in the computational effort. However even in this simpler case the computations remain too complicated and very expensive. Most notable among these methods are the fast free wake method (FFWM) of Miller [1983] and the simplified free wake method (SFWM) of Afjeh and Keith [1986].

This paper presents the wind turbine performance calculations by the lifting-line method. The method is mainly of a prescribed wake type. For a rotating blade, the following principles are applied. First, a blade surface is replaced by a lifting line with the bound circulation varying along its span. The discrete model is used, where the blade is divided into many segments, each segment represented by a helical horseshoe vortex. Second, induced velocities generated by all vortices on the control point of each segment are calculated by the Biot-Savart law. Aerodynamic forces acting on the turbine blade are obtained by the Kutta-Joukowski theorem and using known two-dimensional characteristics of the sectional airfoils. Herein, incompressibility and nonviscosity are assumed throughout the flow field. Unlike methods which assume that the blade wake is a uniform helical surface whose pitch is constant both in the radial and helical directions, the present analysis takes into account the local pitch of each trailing vortex springing from the blade. It is assumed that each element of these trailing vortices moves in space in helical motion, having the pitch calculated with the induced velocity at the point where this element sprang from. Since the wake model is dependent on the induced velocity at each radial station, while this induced velocity is a function of the wake model, the problem becomes nonlinear and is solved using an iterative procedure. The approach may be attractive due to its low computing time and costs. This method of calculation is termed a free helical vortex method (FHVM) and can be applied for the optimal design of an wind turbine blade.

\section{LIFTING LINE METHOD}

\section{Basic Assumptions}

The following assumptions are adopted:

(1) A uniform stream flows parallel to the rotating axis of the wind turbine and the fluid motion is in a stationary state.

(2) Being a straight lifting surface with high aspect ratio, each blade is replaced by a lifting line which is positioned at a quarter chord behind the leading edge and has a varying circulation $\Gamma$ along its span.

(3) Any blade section is considered to work under two-dimensional flow conditions when the complete influence of the induced, rotational and axial velocities on the flow field is taken into account (strip theory). This assumption means that the induced radial velocity is neglected. 
(4) Viscous effects are taken into account only in the two-dimensional properties of each cross section.

(5) Coning and elastic displacements of the blades are neglected and it assumed that the blades remain straight and lie in the rotational plane.

(6) Each element of the trailing vortices which spring from the blade moves freely in space (unconstrained to lie on a surface) in a helical motion whose pitch is constant along the axial direction and equals the pitch at the point where this element shed from. This assumption means that the contraction of the wake is neglected.

\section{Rotor Geometry}

The rotor has a radius $\mathrm{R}$ and it contains B equally spaced blades. All the blades are identical and the chord (c), pitch angle $(\beta)$ and aerodynamic characteristics along the blades are known. In addition, the rotor rotational speed $(\Omega)$ and free stream velocity $\left(V_{0}\right)$ are also known. As shown in Fig. 1 an $(x, y, z)$ Cartesian coordinate system originates from the center of the hub. The axis system is defined by taking an $x$ axis through the quarter chord line of the blade, a $z$ axis pointing to the positive downwind direction, and a $y$ axis that completes the right-hand system. To begin the blade is divided into $\mathrm{N}$ segments. The points of division are denoted

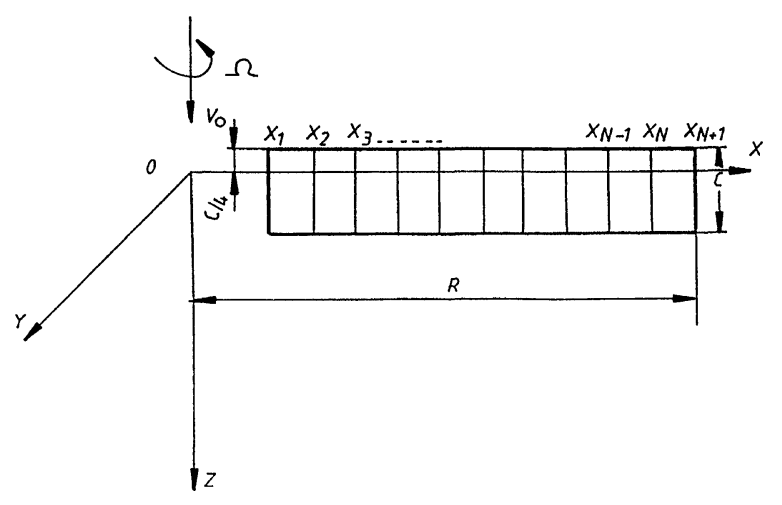

FIGURE 1 Coordinate axes and blade division.

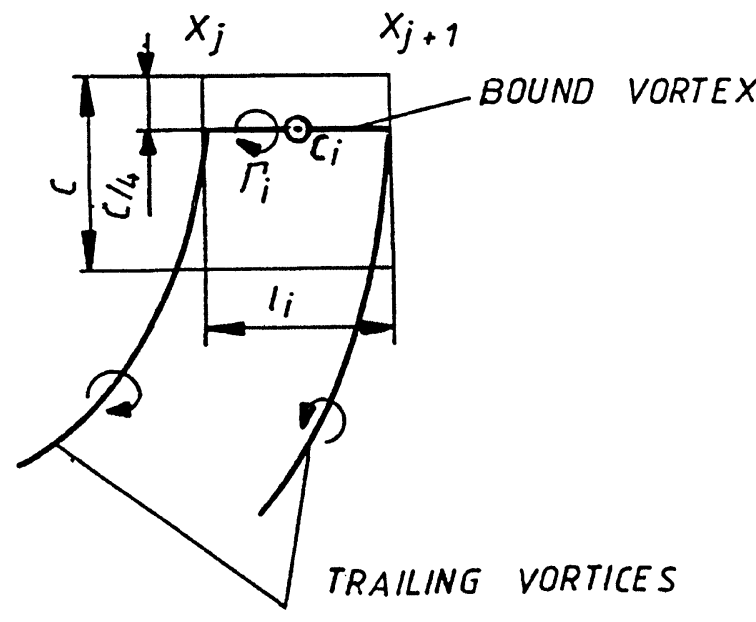

FIGURE 2 Helical horseshoe vortex.

by $x_{j} \quad(j=1,2, \ldots, N+1)$ and it should be noted that this partition may be either uniform or nonuniform. As illustrated in Fig. 2, a bound vortex of constant strength $\Gamma_{i}$ and a control point $C_{i}\left(x_{i}, 0,0\right)$ are put on each segment. The two free vortices shed from both ends of the bound vortex and grow as helical vortices to infinity. The bound vortex and the two trailing helical vortices form a helical horseshoe vortex.

\section{Induced Velocities}

We define $v_{i j}$ and $w_{i j}$ as $(y, z)$ components of induced velocity at the blade itself, at a control point, $\left(x_{i}, 0,0\right)$, and generated by a small helical vortex segment at the point $\left(x_{j}, y_{j}, z_{j}\right)$ on the helical trailing vortex line that springs from the point $\left(x_{j}, 0,0\right)$ with circulation equal to one. If we consider only the axial flow condition, the total normalized induced velocities induced by helical vortex lines (including those from other blades) $V_{i j}, W_{i j}$ can be determined by use of the Biot-Savart law

$$
\begin{aligned}
V_{i j} & =\frac{1}{4 \pi R} \int_{0}^{\infty} v_{i j} \mathrm{~d} \theta, \\
W_{i j} & =\frac{1}{4 \pi R} \int_{0}^{\infty} w_{i j} \mathrm{~d} \theta,
\end{aligned}
$$




$$
\begin{aligned}
& v_{i j}=\sum_{n=1}^{B} \frac{\nu\left[-\eta\left(\cos \theta^{\prime}+\theta \sin \theta^{\prime}\right)+r\right]}{\left[\nu^{2} \theta^{2}+\eta^{2}+r^{2}-2 r \eta \cos \theta^{\prime}\right]^{3 / 2}}, \\
& w_{i j}=\sum_{n=1}^{B} \frac{\eta^{2}-r \eta \cos \theta^{\prime}}{\left[\nu^{2} \theta^{2}+\eta^{2}+r^{2}-2 r \eta \cos \theta^{\prime}\right]^{3 / 2}},
\end{aligned}
$$

where

$$
\eta=\frac{x_{j}}{R}, \quad r=\frac{x_{i}}{R}, \quad \theta^{\prime}=\theta+\frac{2 \pi(n-1)}{B}
$$

$\theta \in[0, \infty)$, is angle of turning of the blade, and

$$
v=\frac{V_{\infty}+w_{j}}{R\left(\Omega+\left(v_{j} / x_{j}\right)\right)} .
$$

We note here that $\left(v_{j}, w_{j}\right)$ are circumferential and axial induced velocities at the radial location of the point of departure of this vortex element.

If the following nondimensional terms are defined

$$
\bar{\Gamma}_{i}=\frac{\Gamma_{i}}{R^{2} \Omega}, \quad \bar{v}_{i}=\frac{v_{i}}{R \Omega}, \quad \bar{w}_{i}=\frac{w_{i}}{R \Omega}
$$

then according to Eqs. (1-4) all B horseshoe vortices $j$ of the B blades induce the nondimensional velocities $\bar{V}_{i j} \bar{\Gamma}_{j}$ and $\bar{W}_{i j} \bar{\Gamma}_{j}$ at the control point $x_{i}$, where the influence coefficients $\bar{V}_{i j}$ and $\bar{W}_{i j}$ are expressed as

$$
\begin{gathered}
\bar{V}_{i j}=\frac{1}{4 \pi} \int_{0}^{\infty}\left(v_{i, j+1}-v_{i, j}\right) \mathrm{d} \theta, \\
\bar{W}_{i j}=\frac{1}{4 \pi} \int_{0}^{\infty}\left(w_{i, j+1}-w_{i, j}\right) \mathrm{d} \theta,
\end{gathered}
$$

Therefore $\bar{v}_{i}$ and $\bar{w}_{i}$, the total induced velocities at the control point $x_{i}$, will be the sum of the contributions of all the horseshoe vortices, and are given by

$$
\bar{v}_{i}=\sum_{j=1}^{N} \bar{V}_{i j} \bar{\Gamma}_{j}, \quad \bar{w}_{i}=\sum_{j=1}^{N} \bar{W}_{i j} \bar{\Gamma}_{j} .
$$

The calculations of $\bar{V}_{i j}$ and $\bar{W}_{i j}$ for different values of $\eta, \mathrm{r}$ and $v$ have been performed using a mixed numerical-analytic solution method [7].
According to this method, the numerical integration is carried only to a certain azimuth angle. Beyond this azimuth angle (to infinity), the integral is evaluated from the average of two analytic integrals that form the lower and upper bounds of the real integrand.

Based on the second assumption and because of symmetry, the bound vortices of the lifting lines themselves do not induce any additional velocities along the blades.

\section{Circulation Distribution and Forces Acting on the Rotor}

We can obtain the lifting forces acting on the bound vortices by the Kutta-Joukowski theorem as follows where $\rho$ is air density and $\mathbf{W}_{i}$ the resultant velocity at $x_{i}$, the middle of the bound vortex $\mathbf{I}_{i}$. In addition, according to the third assumption $\Gamma_{i} \mathbf{l}_{i}$ is orthogonal to the direction of $\mathbf{W}_{i}$ and $\mathbf{L}_{i}$ is also given by

$$
\begin{aligned}
& \mathbf{L}_{i}=\rho \mathbf{W}_{i} \times \Gamma_{i} \mathbf{l}_{i}, \\
& L_{i}=\frac{1}{2} \rho W_{i}^{2} c_{i} k_{i} \alpha_{i} l_{i},
\end{aligned}
$$

where $c_{i}$ is the local chord (with nondimensional form $\left.\bar{c}_{i}=c_{i} / R\right), \alpha_{i}$ is the effective angle of attack and $k_{i}$ is defined by

$$
k_{i}=\frac{C_{L_{i}}\left(\mathrm{Re}_{i}, \alpha_{i}\right)}{\alpha_{i}} .
$$

$C_{L_{i}}$ is the local lift coefficient and is not necessarily a linear function of $\alpha_{i}$. Quantity $\overline{C_{L i}}$ is obtained from the two-dimensional properties of a certain airfoil and is a function of the local Reynolds number $\mathrm{Re}_{i}$ and the effective angle of attack $\alpha_{i}$.

The Reynolds number is defined as

$$
\operatorname{Re}_{i}=\frac{W_{i} c_{i}}{\nu_{a}},
$$

where $\nu_{a}$ is the air kinematic viscosity.

Figure 3 shows the flow at a certain cross section of the blade. It is clear that

$$
\alpha_{i}=\alpha_{G_{i}}-\alpha_{I_{i}},
$$




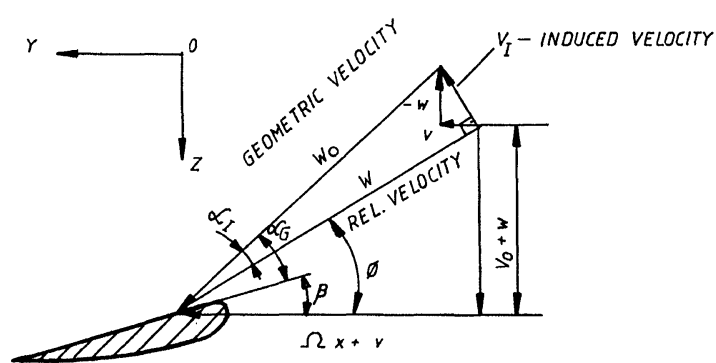

FIGURE 3 Velocity components at the blade cross section.

where $\alpha_{G_{i}}$ is the geometric angle of attack and denotes the angle between the geometric velocity $\mathbf{W}_{O_{i}}$ and the reference axis of the blade cross section,

$$
\alpha_{G_{i}}=\tan ^{-1}\left(\frac{V_{0}}{x_{i} \Omega}\right)-\beta_{i}
$$

The induced angle of attack, $\alpha_{I_{i}}$, is given by

$$
\alpha_{I_{i}}=\frac{V_{I_{i}}}{W_{i}} f_{i}=\frac{w_{i}}{W_{i}} f_{i} \sqrt{1+\left(\frac{v_{i}}{w_{i}}\right)^{2}}
$$

$f_{i}$ is usually a number close to unity, but does not equal unity because $\alpha_{I_{i}}$ is not always a small angle. Quantity $f_{i}$ is obtained from previous iteration.

The substitution of Eq. (14) into (12) and then into Eq. (9), and equating to Eq. (8), results in the following equation

$$
\begin{aligned}
& \frac{2}{\bar{c}_{i} k_{i} f_{i}} \bar{\Gamma}_{i}+\sum_{j=1}^{N} \bar{W}_{i j} \bar{\Gamma}_{j} \sqrt{1+\left(\frac{\bar{v}_{i}}{\bar{w}_{i}}\right)^{2}}=\frac{\alpha_{G_{i}} \bar{W}_{i}}{f_{i}}, \\
& \quad i=1,2, \ldots, N .
\end{aligned}
$$

This is the discrete formulation of the integrodifferential equation of the lifting line method. The above equation (15) is, in fact, a system of simultaneous nonlinear equations with $\bar{\Gamma}_{i}$ unknowns. The nonlinearity of the system is given by all the terms of Eqs. (15) which are functions of the induced velocities at the control points along the blade. We can obtain the circulation distributions along the blade by solving these equations iteratively. Then, we can also calculate axial force, torque and power per blade by summing the axial force, torque and power components over all of the segments. Therefore, the overal axial force, torque and power of the wind turbine rotor are given by

$$
F=\int_{x_{\text {hub }}}^{x_{t i p}} 1 / 2 \rho B c W^{2}\left(C_{L} \cos \Phi+C_{D} \sin \Phi\right) \mathrm{d} x,
$$

$$
Q=\int_{x_{h u b}}^{x_{t i p}} 1 / 2 \rho B c W^{2}\left(C_{L} \sin \Phi-C_{D} \cos \Phi\right) x \mathrm{~d} x,
$$

$P=\int_{x_{h u b}}^{x_{t i p}} 1 / 2 \rho B c W^{2}\left(C_{L} \sin \Phi-C_{D} \cos \Phi\right) x \Omega \mathrm{d} x$,

where $\Phi$ is the relative flow angle.

The axial force, torque and power coefficients are defined as nondimensional parameters of the wind turbine as follows

$$
\begin{gathered}
C_{F}=\frac{F}{1 / 2 \rho \pi R^{2} V_{0}^{2}}, \quad C_{Q}=\frac{Q}{1 / 2 \rho \pi R^{3} V_{0}^{2}}, \\
C_{P}=\frac{P}{1 / 2 \rho \pi R^{2} V_{0}^{3}} .
\end{gathered}
$$

\section{NUMERICAL RESULTS AND DISCUSSION}

In this section, the preceding method is applied to four kinds of rotor configurations, and the calculated results are compared with the existing numerical data of two free wake methods: the FWM of Gohard [1978] and the SFWM of Afjeh and Keith [1986]. Two prescribed wake models are analysed herein: the regular case where the trailing vortices are constrained to distribute along the geometric helical surface described by rotating blade, and another less constrained, where each trailing vortex sheding from the blade forms a free helical line whose pitch depend on the induced velocity at the point where it sprang from. Theoretical results based on these two wake models: geometric helical surface model (GHSM) and free helical vortex model (FHVM), seem to give the upper and lower 
bounds of the real results. Probably the simplest way to calculate the rotor performance is the average of results obtained with these two models.

The blade geometries and operating conditions are presented in Table I. All the cases include twobladed wind turbine rotors with untwisted and constant chord blades.

The airfoil data used in the calculations are given in analytic form as:

$$
\begin{aligned}
& \text { if } \alpha<\alpha_{s}=0.2 \operatorname{rad}\left(11.45^{\circ}\right) \\
& \quad C_{L}=2 \pi \alpha, \quad C_{D}=0.01+0.5 \alpha^{2}
\end{aligned}
$$

$$
\text { if } \begin{aligned}
\alpha \geq \alpha_{s} \\
\quad C_{L}=2 \pi \alpha_{s}, \quad C_{D}=0.01+0.5 \alpha^{2} .
\end{aligned}
$$

The blade is divided into eleven elements in the following way: $\eta_{j}=0.20 ; 0.30 ; 0.40 ; 0.50 ; 0.60 ; 0.70$; $0.75 ; 0.85 ; 0.90 ; 0.95 ; 1.00$ and the control points are located at the center of each segment.

This particular set of rotor configurations and airfoil data were chosen in order to check the validity of the present method by direct comparisons with the theoretical results obtained by more involved methods. For comparison it is given herein the same quantities that have been presented in Afjeh and Keith [1986]. These include distributions along the blade for three aerodynamic quantities (circulation, axial induced velocity and effective angle of attack), only for cases 2 and 4, and overall results (axial force and power coefficients) for all the cases. Figures 4-9 present the aerodynamic quantity distributions along the blade, obtained by the present method, against those of Gohard [1978] and Afjeh and Keith [1986]. It is seen that the

TABLE I Blade geometry and operating conditions for numerical comparisons

\begin{tabular}{lcccc}
\hline Case & $\begin{array}{c}\text { Solidity } \\
B C / \pi R\end{array}$ & $\begin{array}{c}\text { Tip-Speed } \\
\text { ratio } V_{0} / R \Omega\end{array}$ & $\begin{array}{c}\text { Pitch angle } \\
\text { degrees } \beta\end{array}$ & $\begin{array}{c}\text { Chord } \\
\text { distribution }\end{array}$ \\
\hline 1 & 0.106 & 0.105 & 2 & constant \\
2 & 0.106 & 0.154 & 0 & constant \\
3 & 0.106 & 0.154 & 2 & constant \\
4 & 0.106 & 0.154 & 4 & constant \\
\hline
\end{tabular}

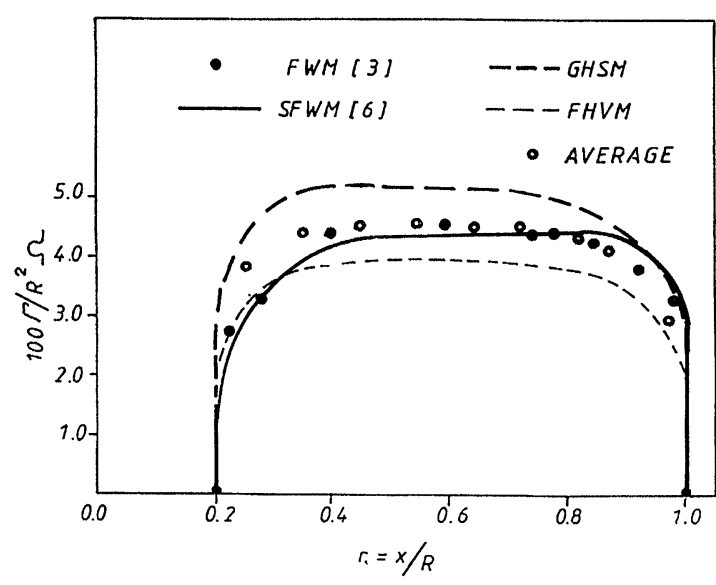

FIGURE 4 Computed nondimensional circulation distribution along the blade for case 2 conditions, Table I.

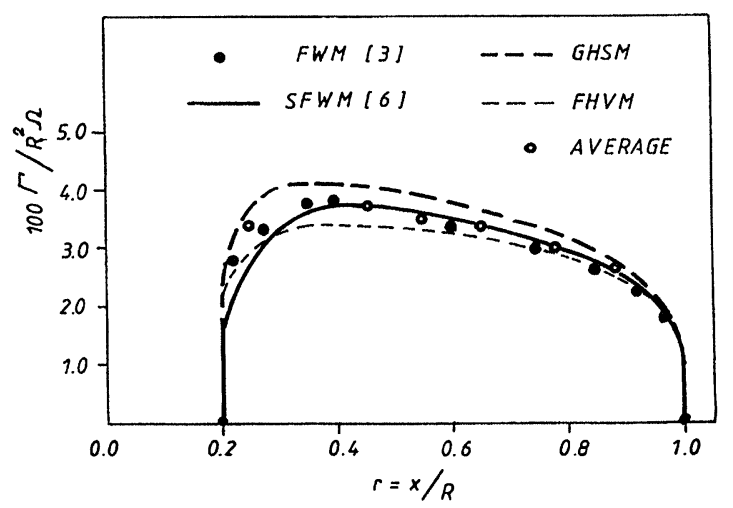

FIGURE 5 Computed nondimensional circulation distribution along the blade for case 4 conditions, Table I.

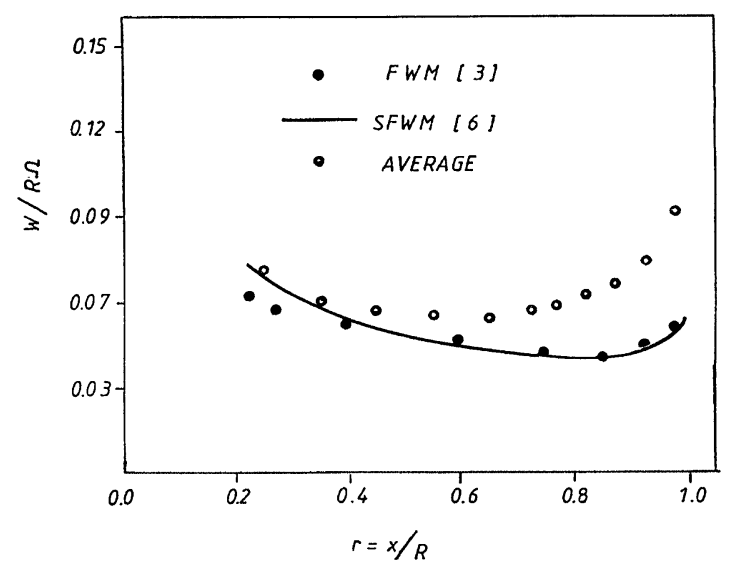

FIGURE 6 Computed spanwise axial induced velocity distribution for case 2 conditions, Table I. 
present computed results, evaluated as simple average of two bound results, agree favorably with those of the test methods. The differences are attributed to the different modelling of the trailing vortices.

The total integrated axial force and power coefficients calculated by different methods of analysis are contained in Table II. It is seen that the results (as average values) of the present approach agree well with the predictions of the two test methods. It seems that as value of tipspeed ratio (tangential over free stream velocity) increases (case 1) the wake deformation effects become dominant, and probably a weighted mean of bound results with inverse tip-speed ratio, $\nu_{0}=V_{0} / R \Omega$, as weighted function, should be more suitable. The other comparisons are necessary to validate this new point of view.

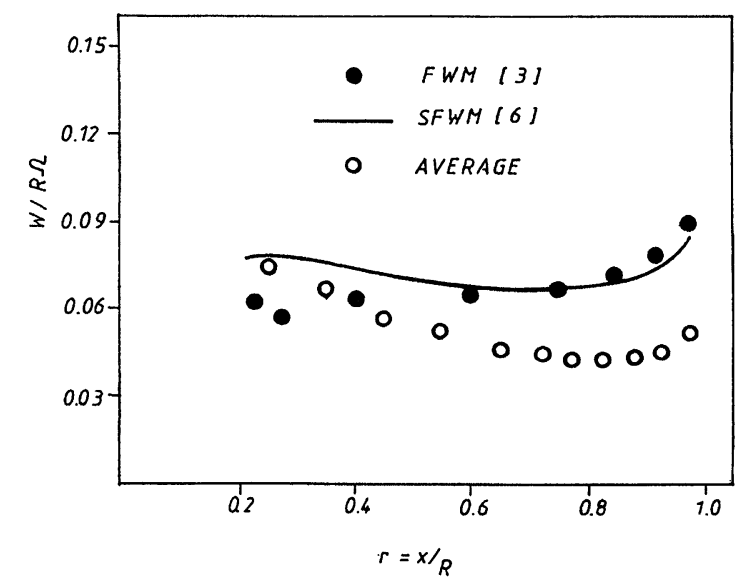

FIGURE 7 Computed spanwise axial induced velocity distribution for case 4 conditions, Table I.

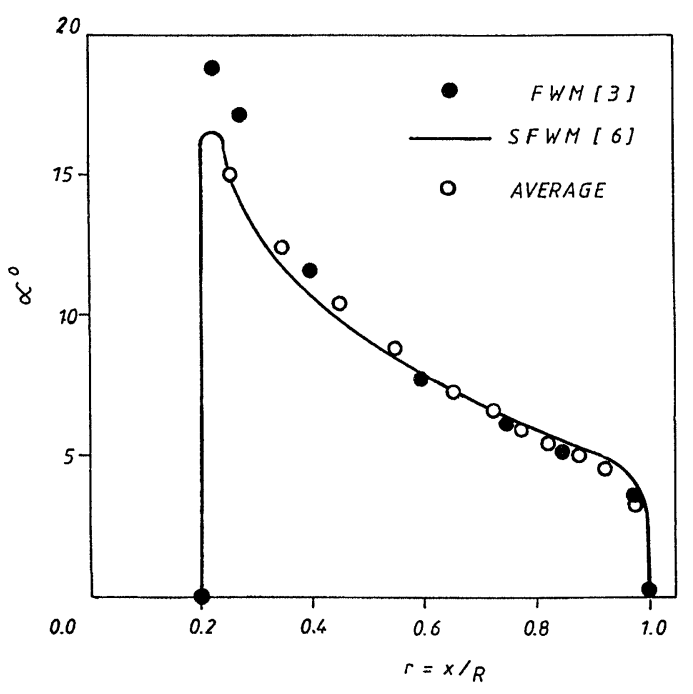

FIGURE 8 Computed effective angle of attack distribution along the blade for case 2 conditions, Table I.

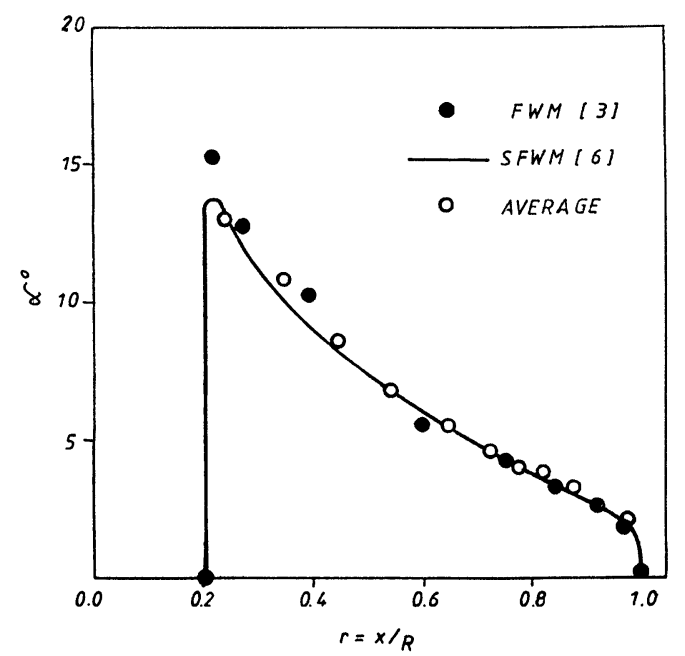

FIGURE 9 Computed effective angle of attack distribution along the blade for case 4 conditions, Table I.

TABLE II Comparison of power and axial force coefficients

\begin{tabular}{cccccccc}
\hline & Case & FWM $[3]$ & SFWM $[6]$ & GHSM & FHVM & Average & Weighted mean \\
\hline CP & 1 & 0.3489 & 0.417 & 0.51560 & 0.28167 & 0.39863 & 0.30623 \\
& 2 & 0.4575 & 0.454 & 0.57296 & 0.32014 & 0.44655 & 0.35907 \\
& 3 & 0.4609 & 0.473 & 0.55362 & 0.39260 & 0.47311 & 0.41740 \\
$\mathrm{CF}$ & 4 & 0.4379 & 0.451 & 0.50165 & 0.39919 & 0.45042 & 0.41497 \\
& 1 & 1.0762 & 1.144 & 1.26222 & 0.99104 & 1.12663 & 1.01951 \\
& 2 & 1.1145 & 1.103 & 1.22614 & 0.95713 & 1.09164 & 0.99856 \\
& 3 & 0.9527 & 0.959 & 1.04504 & 0.87943 & 0.96224 & 0.90493 \\
& 4 & 0.7964 & 0.803 & 0.86107 & 0.75555 & 0.80831 & 0.77180 \\
\hline
\end{tabular}


On the other hand, the present approach offers definite computer time savings and can be useful as a design tool.

\section{CONCLUSIONS}

The nonlinear iterative prescribed wake analysis which has been presented in this paper seem to give good results in comparison with free wake methods. Theoretical results based on two very simple wake models seem to give the upper and lower bounds of the real results. The theoretical aerodynamic loads and performance of a number of rotors has been calculated as the average of these bound values. For relative high values of tip-speed ratio where wake deformation effects are dominant a weighted mean of bound values seems to be more suitable. The inverse tip-speed ratio can be used as weighted function.

The advantage of the present approach - except for being quite efficient as computing time and accuracy - is that the present prescribed wake model is determined according to simple physical reasoning. There is no need for different parameters which define the wake structure and which are determined according to previous empirical experience. Such empirical experience is always limited, and its application to cases where this experimental evidence does not exist is usually accompanied by severe doubts. Accordingly, this method of calculation can be applied for the optimal design of a wind turbine blade.

\section{NOMENCLATURE}

$$
\begin{aligned}
& B=\text { number of blades } \\
& c=\text { airfoil section cord } \\
& C_{D}=\text { drag coefficient } \\
& C_{F}=\text { axial force thrust coefficient } \\
& C_{i}=\text { control point, refer to Fig. } 2 \\
& C_{L}=\text { lift coefficient } \\
& C_{Q}=\text { torque coefficient } \\
& C_{P}=\text { power coefficient } \\
& F=\text { axial force on wind turbine }
\end{aligned}
$$

$f_{i} \quad=$ correction factor in Eq. (14)

$k_{i} \quad=$ quantity defined by Eq. (10)

$l_{i} \quad=$ length of bound vortex element (m)

$L_{i} \quad=$ bound vortex lift $(\mathrm{N})$

$P \quad=$ power $(\mathrm{W})$

$Q \quad=$ torque $(\mathrm{Nm})$

$r \quad=$ nondimensional distance along the blade

$R \quad=$ radius of blade $(\mathrm{m})$

$\mathrm{Re} \quad=$ Reynolds number

$v_{i}, w_{i}=\mathbf{y}, \mathbf{z}$ components of total induced velocity at the control point $\mathrm{I}(\mathrm{m} / \mathrm{s})$

$V_{i j}, W_{i j}=\mathbf{y}, \mathbf{z}$ components of induced velocity by helical vortex filaments of unit strength $(\mathrm{m} / \mathrm{s})$

$\bar{V}_{i j}, \bar{W}_{i j}=\mathbf{y}, \mathbf{z}$ influence coefficients

$V_{0} \quad=$ wind velocity $(\mathrm{m} / \mathrm{s})$

$\mathbf{W} \quad=$ resultant velocity $(\mathrm{m} / \mathrm{s})$

$\mathbf{x}, \mathbf{y}, \mathbf{z}=$ rectangular coordinates of point, refer to Fig. $1(\mathrm{~m})$

$x_{i}, 0,0=$ coordinates of the control point $(\mathrm{m})$

$x_{j}, 0,0=$ coordinates of the starting point of the trailing vortex filament $(\mathrm{m})$

\section{Greek Symbols}

$\alpha=$ angle of attack ( $\mathrm{rad})$

$\alpha_{G}=$ geometric angle of attack (rad)

$\alpha_{I}=$ induced angle of attack (rad)

$\alpha_{s}=$ stall angle of attack (rad)

$\beta=$ section blade pitch angle ( $\mathrm{rad})$

$\Gamma=$ circulation $\left(\mathrm{m}^{2} / \mathrm{s}\right)$

$\Phi=$ flow angle (rad)

$\eta=$ nondimensional radius of the trailing vortex filament

$\nu=$ pitch of the helical vortex filament

$\nu_{a}=$ kinematic viscosity $\left(\mathrm{m}^{2} / \mathrm{s}\right)$

$\rho=$ density of fluid $\left(\mathrm{Kg} / \mathrm{m}^{3}\right)$

$\theta=$ angle of turning of the blade (rad)

$\theta^{\prime}=\theta+2 \pi(n-1) / B(\mathrm{rad})$

$\Omega=$ rotational speed $(\mathrm{rad} / \mathrm{s})$

\section{References}

Afjeh, A.A., Keith, T.G. (1986) A Simplified Free Wake Method for Horizontal-Axis Wind Turbine Performance Prediction, Journal of Fluids Engineering, Vol. 108, pp. 400-406. 
Chiu, Y.D., Peters, D.A. (1988) Numerical Solutions of Induced Velocities by Semi-Infinite Tip Vortex Lines, Journal of Aircraft, Vol. 25, pp. 684-694.

De Vries, O. (1979) Fluid Dynamic Aspects of Wind Energy Conversion, AGARDograph No. 243.

De Vries, O. (1983) On the Theory of Horizontal-Axis Wind Turbines, Annual Review of Fluid Mechanics, Vol. 15, pp. $77-96$.

Gohard, J.C. (1978) Free Wake Analysis of Wind Turbine, Aerodynamics, ASRL TR-184-14, Aero. and Struc. Research Lab., Dept of Aeronautics and Astronautics, MIT.
Maekawa, H. (1984) Application of the Vortex Theory to HighSpeed Horizontal-Axis Wind Turbines, Bulletin of JSME, vol. 27, No. 229, pp. 1460-1466.

Miller, R.H. (1983) The Aerodynamics and Dynamic Analysis of Horizontal-Axis Wind Turbines, Journal of Wind Engineering and Industrial Aerodynamics, Vol. 15, pp. 329-340. 


\section{ait \\ ENERGY MATERIALS}

M A N E Y publishing

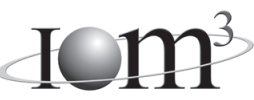

\section{Materials Science \& Engineering for Energy Systems}

Maney Publishing on behalf of the Institute of Materials, Minerals and Mining

The Institute of Materials, Minerals \& Mining

Economic and environmental factors are creating ever greater pressures for the efficient generation, transmission and use of energy. Materials developments are crucial to progress in all these areas: to innovation in design; to extending lifetime and maintenance intervals; and to successful operation in more demanding environments. Drawing together the broad community with interests in these areas, Energy Materials addresses materials needs in future energy generation, transmission, utilisation, conservation and storage. The journal covers thermal generation and gas turbines; renewable power (wind, wave, tidal, hydro, solar and geothermal); fuel cells (low and high temperature); materials issues relevant to biomass and biotechnology; nuclear power generation (fission and fusion); hydrogen generation and storage in the context of the 'hydrogen economy'; and the transmission and storage of the energy produced.

As well as publishing high-quality peer-reviewed research, Energy Materials promotes discussion of issues common to all sectors, through commissioned reviews and commentaries. The journal includes coverage of energy economics and policy, and broader social issues, since the political and legislative context influence research and investment decisions.

\section{CALL FOR PAPERS}

Contributions to the journal should be submitted online at http://ema.edmgr.com

To view the Notes for Contributors please visit: www.maney.co.uk/journals/notes/ema

Upon publication in 2006, this journal will be available via the Ingenta Connect journals service. To view free sample content online visit: www.ingentaconnect.com/content/maney

For further information please contact:

Maney Publishing UK

Tel: +44 (0)113 2497481 Fax: +44 (0)1132486983 Email: subscriptions@maney.co.uk

or

Maney Publishing North America

Tel (toll free): 8662975154 Fax: 6173546875 Email: maney@maneyusa.com

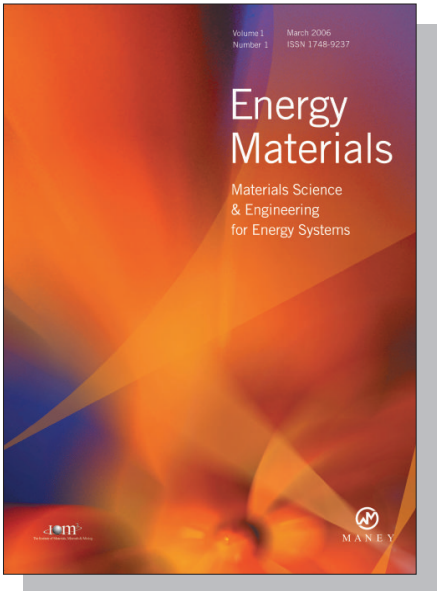

EDITORS

Dr Fujio Abe

NIMS, Japan

Dr John Hald, IPL-MPT, Technical University of Denmark, Denmark

Dr R Viswanathan, EPRI, USA

\section{SUBSCRIPTION INFORMATION}

Volume 1 (2006), 4 issues per year

Print ISSN: 1748-9237 Online ISSN: 1748-9245

Individual rate: $£ 76.00 / U S \$ 141.00$

Institutional rate: $£ 235.00 /$ US $\$ 435.00$

Online-only institutional rate: $£ 199.00 / U S \$ 367.00$

For special $\mathrm{IOM}^{3}$ member rates please email

subscriptions@maney.co.uk

\section{For further information or to subscribe online please visit www.maney.co.uk}



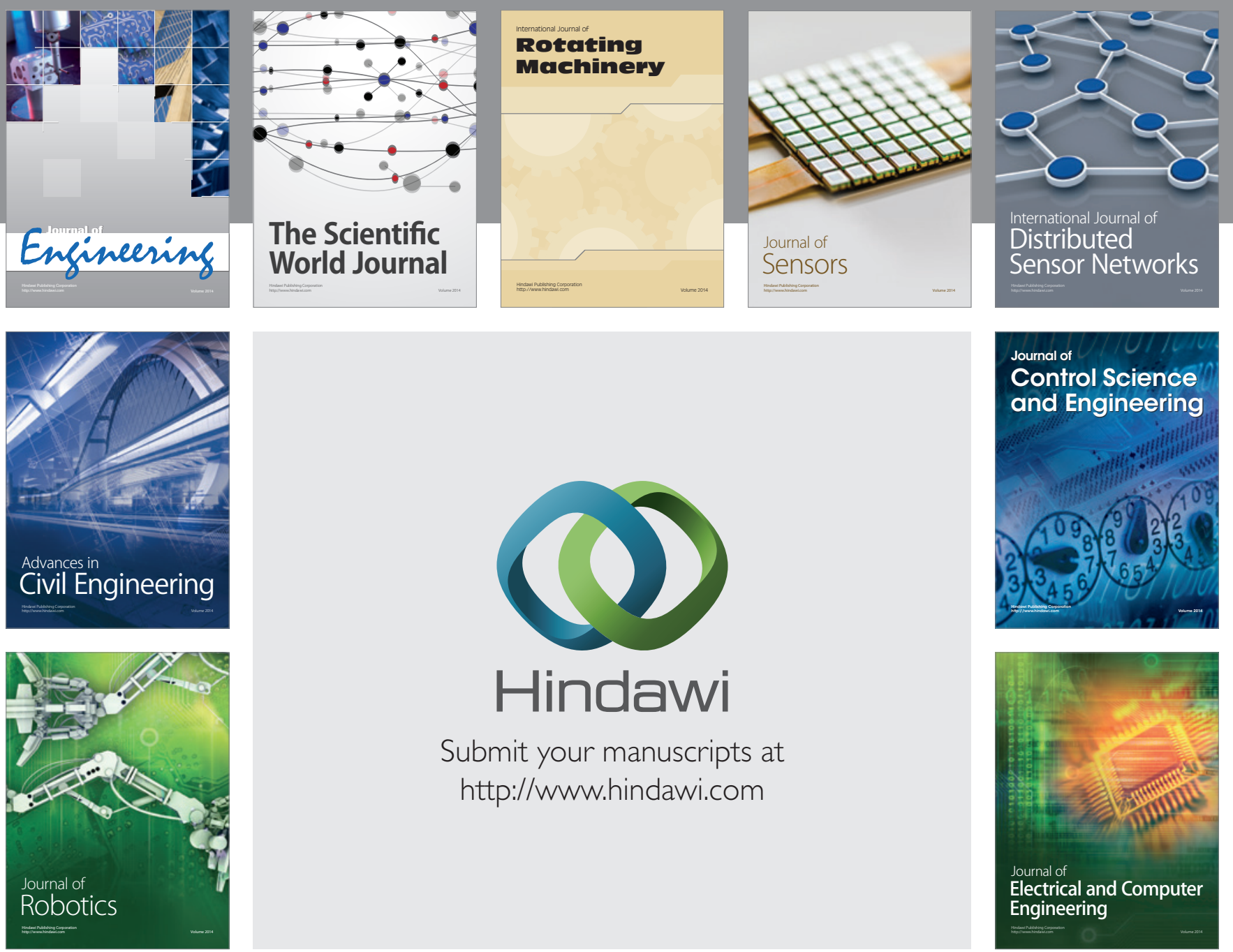

Submit your manuscripts at

http://www.hindawi.com
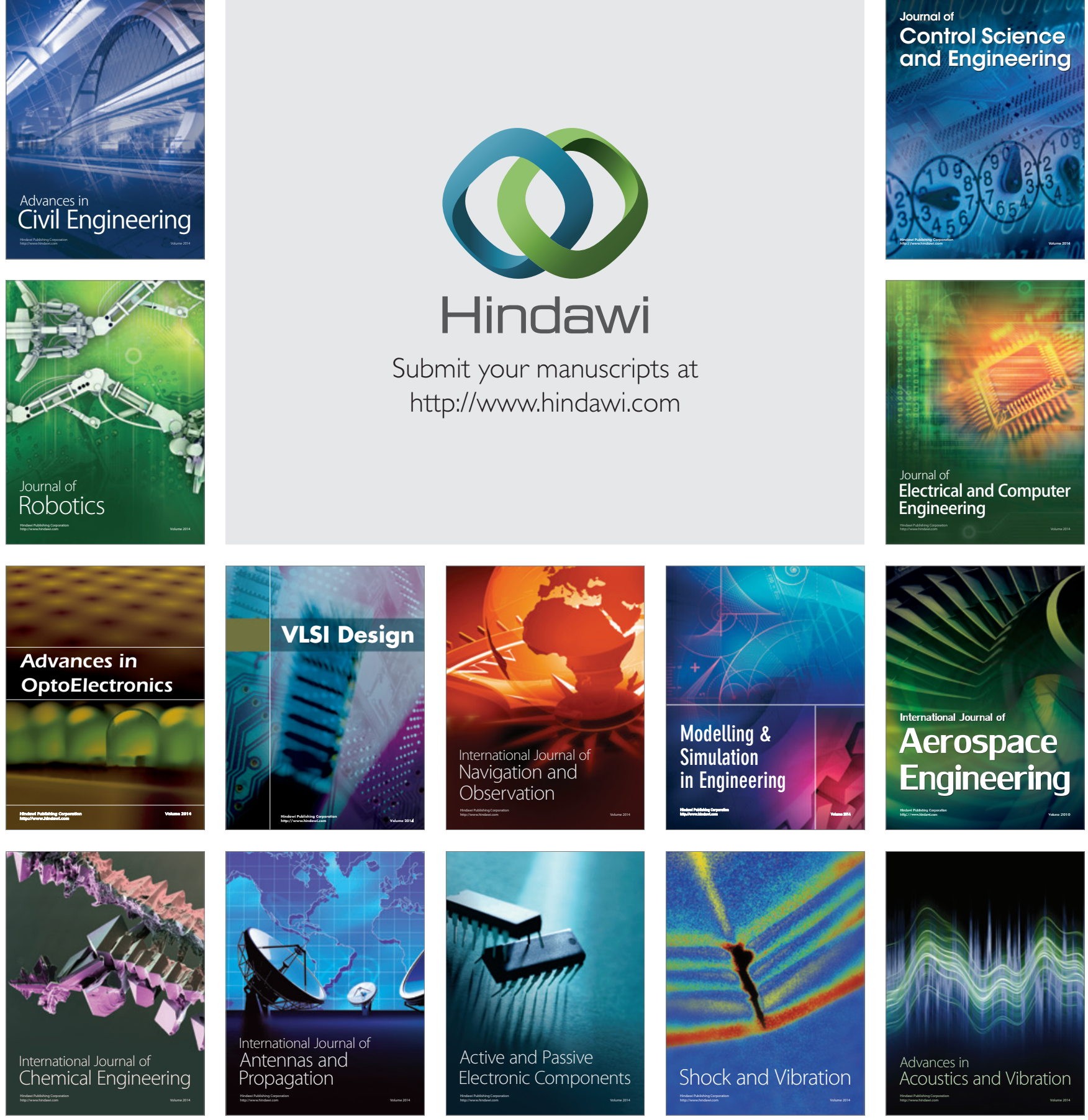